\title{
Future Directions for Radial-Velocity Research
}

\author{
David W. Latham \\ Harvard-Smithsonian Center for Astrophysics, 60 Garden Street, \\ Cambridge, Massachusetts, 02138
}

\begin{abstract}
.
I review the status of ground-based radial-velocity searches for extrasolar planets and speculate about the new results that can be expected in this field over the coming years. Then I review the plans for astrometric space missions and speculate about the impact that these missions will have on ground-based radial-velocity work, citing the specific examples of extra-solar planet research, the mass-luminosity relation for $M$ dwarfs and metal-poor stars, and Galactic structure and evolution.
\end{abstract}

\section{Introduction}

It is always a daunting challenge to forecast the future, so it was with some misgivings that I accepted the assignment to speak on the future of stellar radial-velocity research. I decided to ignore the issue of future technological developments in the measurement of precise stellar radial velocities, and to focus instead on the research directions that I thought would prove most fruitful over the coming years.

Over the near-term future $I$ foresee that the highest-profile radial-velocity research will be the search for and characterization of extrasolar planets. Undoubtedly there will also be progress in various other areas, such as studies of stellar activity and the determination of accurate masses, but in this paper I restrict my prognostication to the lines of extrasolar planet research that look the most promising to me.

Over the longer term I believe that the future of stellar radial-velocity research will become intimately connected with astrometric space missions such as DIVA, FAME, GAIA, and SIM. Here again I expect that extrasolar planet research will have the highest profile, but other areas of astrophysics, such as the determination of very accurate masses for low-mass stars, and the study of Galactic structure and evolution, will also be important.

\section{Ground-Based Radial-Velocity Extrasolar Planet Research}

In this section I discuss the most promising directions that I foresee for extrasolar planet research using precise radial velocities. We are already in a reconnaissance phase, where the velocities of more than a thousand solar-type stars are being monitored with a precision of $10 \mathrm{~m} \mathrm{~s}^{-1}$ or better by several different teams. Over the next decade we can expect these surveys to uncover several dozen new giant 


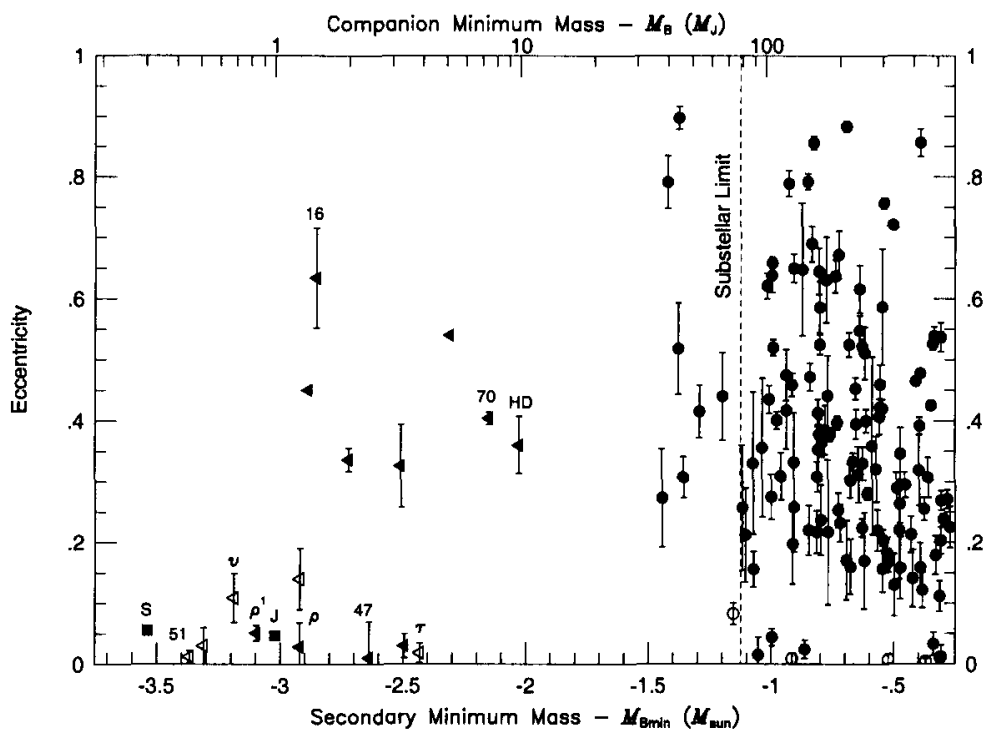

Figure 1. Eccentricity versus the $\log$ of minimum companion mass for 16 radial-velocity planet candidates (triangles), together with Jupiter and Saturn (squares) and 132 of the low-mass secondaries (circles) found in the Carney-Latham proper-motion sample.

planets. This should help clarify the overall characteristics of the population of giant planets orbiting solar-type stars. These characteristics are now just beginning to emerge and are poorly defined because of the small number of orbits determined so far.

To illustrate the orbital characteristics of the planets known now, in Fig. 1 I plot eccentricity versus the log of minimum companion mass for the 16 planet candidates (triangles) announced as of October 1998 (Schneider 1998, Marcy \& Butler 1998), together with Jupiter and Saturn (squares) and 132 of the lowmass secondaries (circles) found in the Carney-Latham proper-motion sample (Latham et al. 1999, Carney et al. 1994). The planet candidates with published orbits are labelled with abbreviations, while the ones that have only been announced at meetings or via the World Wide Web are unlabelled. Systems with orbital periods shorter than 13 days have been plotted with open symbols. The orbits of these systems have presumably been circularized by tidal mechanisms, so there is no record of the orbital eccentricity they formed with.

The stellar companions concentrate on the right side of Fig. 1, with the vast majority of the minimum masses falling above the substellar limit near $75 M_{J}$, shown as the vertical dashed line. These binary orbits show a wide distribution of orbital eccentricities, as might be expected for stellar companions that formed by the infall of material during the gravitational collapse of a protostellar cloud.

Below a minimum mass of about $10 M_{J}$, there appear to be two populations. One has a distribution of orbital eccentricities similar to that of the low-mass stellar companions ( 7 objects), while the other shows only circular (or nearly circular) orbits. 
The traditional view of how planets must form was heavily influenced by what we knew about our own solar system. It was usually assumed that giant planets would naturally form with circular orbits because of dissipation in the circumstellar disks from which they formed (e.g. Duquennoy \& Mayor 1991). Very few of the planet candidates with circular orbits have periods long enough to prevent circularization of the orbits by tidal mechanisms (e.g. Mathieu, et al. 1992, Marcy et al. 1997), and in just three cases it may be that we are actually seeing the low eccentricity with which they formed.

How about the planet candidates with eccentric orbits? In at least one case, 16 Cygni B, the orbital eccentricity may have been pumped up by tidal interactions with the distant visual companion, 16 Cygni A (Holman, Touma, \& Tremaine 1997; Mazeh, Krymolowski, \& Rosenfeld 1997). But, this mechanism cannot be invoked to explain most of these eccentric orbits, because there is no evidence for a third star in most of the systems. One possible mechanism for inducing some orbital eccentricity might be interactions with massive circumstellar disks during the pre-main-sequence stage (Artymowicz 1992). However, for this mechanism to work the companion must have sufficient mass to excite the necessary resonances in the disk, and it appears unlikely that the smallest planets are massive enough. Another possibility might be that these companions formed like binary stars, by the fragmentation of a collapsing protostellar cloud (e.g. Bodenheimer, Ruzmaikina, \& Mathieu 1993). However, it appears that the lower limit for the mass from fragmentation is much larger than the minimum masses found for the smallest companions in eccentric orbits. Perhaps the most viable candidate for the mechanism responsible for these eccentric orbits is gravitational scattering (Weidenschilling \& Marzari 1996; Rasio \& Ford 1996). The basic idea is that a family of giant planets would form initially, and then gravitational interactions would send one of the planets into an eccentric orbit with small periastron, while the other planets would be scattered into wider orbits or even out of the system altogether.

With the passage of time, as the duration of the observational efforts lengthens, we can hope that some true analogs of Jupiter will be found, giant planets with periods on the order of a decade and orbital amplitudes for the reflex motion of the parent stars on the order of $10 \mathrm{~m} \mathrm{~s}^{-1}$. The companions detected so far all have periods shorter than five years and orbital amplitudes larger than 50 $\mathrm{m} \mathrm{s}^{-1}$, so detecting true analogs to Jupiter will require major progress on both fronts.

How many of the planets discovered so far are accompanied by additional planets, and what are the characteristics of such planetary systems? One strategy for answering these questions will be to monitor the radial velocities of stars known to have a planetary companion, with better precision and longer time coverage, in order to search for additional periodicities. The discovery of additional companions in stable circular (and presumably coplanar) orbits would provide a clinching argument for planetary systems that formed by accretion in circumstellar disks. Moreover, orbital characteristics, such as the periods and spacings, and the relative masses, would illuminate the details of the formation and evolutionary processes. Already there are hints of an additional companion in at least two of the known systems, so the prospects are good that the first extrasolar planetary systems will reveal themselves over the coming decade. 
Another important direction for radial-velocity planet searches will be to push the sensitivity to lower minimum masses. What is the frequency of planets the size of Saturn and Uranus? Do planetary cores an order of magnitude more massive than the earth form closer to their parent stars than the ice condensation zone? Here the emphasis would be on reaching the best possible velocity precision and accumulating rich data sets. The discovery orbit for the unseen companion of HD 114762 (Latham et al. 1989) illustrates that it is feasible to detect and solve orbits where the amplitude is as small as the error in the individual velocity measurements, but it does require dozens of measurements per target.

How do the characteristics of planets and planetary systems depend on the spectral type and mass of the parent star? Does metallicity play a role? To what extent can planetary companions coexist with stellar companions? These are examples of the sort of issues that will receive attention as the population of known planetary companions grows.

\section{The Impact of Astrometric Space Missions}

In this section I summarize the plans for astrometric space missions, and compare their capabilities for extrasolar planet searches. Then I discuss some of the areas where I expect these missions to have an impact on ground-based radial-velocity research.

The remarkable success of the Hipparcos mission has had a strong influence on the plans for future astrometric space missions. DIVA, FAME, and GAIA are all global scanning surveys in the Hipparcos tradition, but with arrays of modern CCD detectors instead of image dissectors, promising enormous gains in the sensitivity limits and numbers of targets. In contrast, SIM is designed to point at individual targets, in order to achieve the best possible astrometric accuracy. As a result, SIM may be able to reach the exquisite accuracy needed to detect terrestrial planets orbiting the nearest solar-type stars.

The High Precision Parallax Collecting Satellite, Hipparcos (1998), pioneered the strategy of a global scanning survey. The Hipparcos aperture was a decent size, $30 \mathrm{~cm}$ in diameter, but the detectors were image dissectors with S20 cathodes. Although the quantum efficiency of modern CCDs is several times better than S20 cathodes, the biggest advantage of CCD arrays is the ability to multiplex on many targets simultaneously, which translates into the ability to reach many more targets and much fainter limits. Hipparcos measured about 120,000 targets with an astrometric mission accuracy of 1 to 2 mas. The target list was reasonably complete down to about $V=9 \mathrm{mag}$, and included targets of special interest down past $V=12$ mag. Hipparcos yielded a gold mine of new astrometric information for stellar binaries. More than 20,000 binary systems were analyzed, including about 3,000 newly-discovered systems. However, the limit to the astrometric precision of 1 to 2 mas meant that Hipparcos was barely able to push below the substellar limit in its search for low-mass companions orbiting nearby solar-type dwarfs.

The Deutsches Interferometer für Vielkanalphotometrie und Astrometrie, DIVA (1998), has been proposed as a modest mission that could extend the successful work of Hipparcos and set the stage for a more ambitious ESA mission. 
The main technological advance would be to use an array of astrometric and photometric CCDs as the detectors. The satellite itself would be quite small, and the two Fizeau interferometers would have only $10 \mathrm{~cm}$ baselines. Nevertheless, the goal would be to analyze at least 10 million stars, and for the half million targets brighter than $V=10.5 \mathrm{mag}$ the astrometric accuracy would be 0.2 mas, almost ten times better than Hipparcos. This performance would open up the entire brown-dwarf regime from 75 to $15 M_{J}$ to the scrutiny of DIVA, but would fall short of reaching $1 M_{J}$ companions.

The Full-Sky Astrometric Mapping Explorer, FAME (1998), has been proposed for a medium-cost mission utilizing a Delta launcher. With a $0.5-\mathrm{m}$ telescope it would reach 40 million stars and would achieve an astrometric accuracy of 0.05 mas for stars brighter than $V=10.5 \mathrm{mag}$. This would give FAME somewhat better sensitivity than DIVA for the detection of planets, but it still would not have much impact on the search for companions as small as $1 M_{J}$.

The Global Astrometric Interferometer for Astrophysics, GAIA (1998), has been proposed as a Cornerstone Mission within the ESA Horizon 2000 Plus science plan, and might fly as early as 2009 . It is a much more ambitious project than either DIVA or FAME. With its two $1.7 \times 0.7-\mathrm{m}$ telescopes and its array of 276 astrometric and photometric CCDs, GAIA would achieve an astrometric accuracy of 0.004 mas down to $V=10.5 \mathrm{mag}$. With this performance GAIA could explore fully the population of Jupiter-mass companions in 1 AU orbits.

The Space Interferometry Mission, SIM (1998), is now under intense study and development and is scheduled for launch in June 2005. SIM will be a pointed instrument, which should allow it to reach better astrometric accuracy on fainter objects than GAIA, at the cost of a much smaller target list. In its wide-angle mode the astrometric accuracy of SIM is no better than GAIA for bright stars. Moreover, GAIA will look at far more targets because it is an all-sky scanning mission, while SIM is a pointed mission. This is why the number of targets that SIM can investigate, even for a major project, is limited to something like one or two thousand. Nevertheless, SIM has the advantage that it will be launched first, and therefore it can undertake the initial astrometric exploration of the 1 $M_{J}$ regime. Perhaps the most exciting prospect for SIM is that in its narrowangle mode it may be able to achieve the exquisite astrometric accuracy needed to detect terrestrial planets orbiting the nearest solar-type stars.

\subsection{Impact on Radial-Velocity Extrasolar Planet Research}

By the time that SIM flies in 2005, we can expect that ground-based radialvelocity searches for extrasolar planets will have discovered several dozen giant planets in orbit around solar-type stars within about $100 \mathrm{pc}$ of the sun. SIM will be able to improve our understanding of the radial-velocity planets in two important ways.

First, SIM will be able to determine the orbital inclination for most of the radial-velocity planets. Even Hot Jupiters, in very short-period tight orbits like the companion of 51 Pegasi, will be measureable by SIM, if it can achieve an astrometric accuracy of $0.1 \mu$ as in its narrow-angle mode. If all we have is a single-lined spectroscopic orbit and an estimate of the primary mass, then the actual mass of the companion is ambiguous, because $\sin i$ is unknown. We can only be sure of the minimum mass, i.e. the mass the companion would have 
for the limiting case when the orbit is viewed edge-on with $\sin i=1$. Knowing the orbital inclination from astrometry will remove this ambiguity and give us companion masses directly, assuming we have good estimates for the primary masses.

Second, SIM will be able to search for additional companions in the systems with radial-velocity planets. Astrometric techniques complement Doppler techniques, because the sensitivity of astrometry to orbital motion improves with period, while the sensitivity of radial velocities decreases with period. The goal would be to learn about the formation and evolution of planetary systems. For example, how many of the Hot Jupiter systems have additional giant planets in long-period orbits? Could these additional planets have been knocked into their wide orbits by gravitational scatterings? And, if true analogs of Jupiter have been discovered by radial velocity searches by the time SIM flies, it will be very interesting to see if they have additional companions like the planets in our own solar system.

\subsection{Impact on the Mass-Luminosity Relation}

Double-lined eclipsing binaries are the main source of very precise stellar masses and radii (e.g. Andersen 1997), contributing more than 100 determinations with better than $2 \%$ precision over the range 0.6 to $20 M_{\odot}$. For main-sequence dwarfs with masses below $0.6 M_{\odot}$, good results have been published for only two double-lined eclipsing systems, YY Gem and CM Dra (Leung \& Schneider 1978, Metcalfe et al. 1996), with an important new result for a third M dwarf eclipsing binary, GJ 2069, announced at this Colloquium (Delfosse et al. 1999).

For eclipsing binaries it is normally possible to derive good values for the luminosities, if suitable photometry is available for deriving a reliable effective temperature, because the stellar radius comes directly out of the orbital solution. However, for cool stars the bolometric corrections are rarely well determined, and the usual practice is to work with absolute magnitudes rather than luminosities. This is the reason I have chosen to use the absolute magnitude in the $V$ band for the vertical axis in Fig. 2, where I plot mass versus absolute $V$ magnitude from about $10 M_{\odot}$ down to the substellar limit near $0.075 M_{\odot}$. Ninety of the points in Fig. 2 are for eclipsing binary masses from Andersen's review (1991), plotted as open circles. The results for eclipsing binaries published since 1991 are plotted as 30 filled circles, adopting the same limit of $2 \%$ for the mass precision.

In most cases the uncertainties are similar to the size of the symbols in Fig. 2 , and the spread among the points for the eclipsing binaries is often larger than the uncertainties due to observational errors. This illustrates a point emphasized by Andersen $(1991,1997)$, that detailed analyses of the individual stars in eclipsing binary systems must include the effects of metallicity, helium abundance, and age, in order to take full advantage of the precision now available in the masses and radii, when testing stellar models and evolution theory. Another important point is that no accurate mass determinations have yet been published for old, metal-poor members of the Galactic halo, which means that we do not yet have any fundamental tests of the masses used for the models of stellar structure and evolution that we rely on for interpreting the oldest stars in the Galaxy, including the globular-cluster stars. 


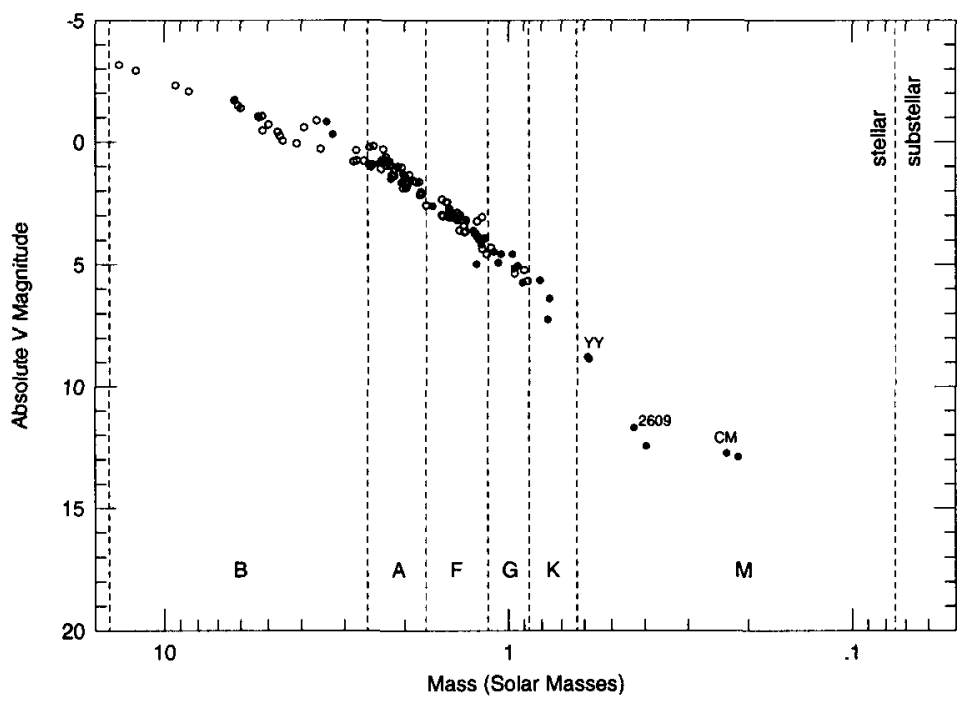

Figure 2. Mass vs. absolute $V$ magnitude for eclipsing binaries with determinations better than $2 \%$.

Astronomy is now entering an era when interferometers should be able to provide excellent astrometric orbits for binaries with much shorter periods than traditionally has been possible. At the same time, new techniques have been developed for deriving double-lined spectroscopic orbits for binaries where the two sets of lines are never well separated (e.g. Andersen 1997). Together these two developments promise a rich harvest of new mass determinations. Thus, interferometers should have a major impact on improving our knowledge of the mass-luminosity relation for $\mathrm{M}$ dwarfs and metal-poor stars, by supplying good orbital inclinations for dozens of double-lined systems with good spectroscopic orbits.

\subsection{Impact on Galactic Structure and Evolution}

The astrometric space missions should have an enormous impact on the study of Galactic structure and evolution. Global-scanning missions such as DIVA, FAME, and GAIA will provide accurate proper motions and parallaxes for literally millions of stars. When combined with accurate radial velocities, this will allow high-quality space motions and Galactic orbits to be derived for large samples of stars with extreme kinematics, such as the halo and thick disk populations. Moreover, the space missions should be able to reach limits faint enough to provide in situ samples, thus removing the difficult observational biases associated with kinematically-selected samples of nearby stars.

The rich harvest of new proper motions and parallaxes from space will pose a daunting challenge to ground-based stellar spectroscopists, not only to provide the radial velocities needed to derive space motions, but also to provide the spectroscopic metallicities needed to study the subtle relations between kinematics and chemistry that can be used to retrace the early history of our 
Galaxy. Although there is some talk about providing GAIA with the capability to measure crude radial velocities for millions of stars, I do not believe that this will satisfy the demand for high-quality spectroscopic observations. This would appear to be an ideal application for ground-based multi-object fiber-fed echelle spectrographs with large-format CCD detectors working in single-order mode.

\section{References}

Andersen, J. 1991, A\&AR, 3, 91

Andersen, J. 1997, in Fundamental Stellar Properties: the Interaction between Observation and Theory (IAU Symp. 189), T.R. Bedding, A.J. booth, \& J. Davis, Dordrecht: Kluwer, 99

Artymowicz, P. 1992, PASP, 104, 769

Bodenheimer, P., Ruzmaikina, T., \& Mathieu, R.D. 1993, in Protostars and Planets III, E.H. Levy \& J.I. Lunine, Tucson: Univ. Arizona Press, 367

Carney, B.W., Latham, D.W., Laird, J.B., \& Aguilar, L.A. 1994, AJ, 107, 2240

Delfosse, X., Forveille, T., Mayor, M., Burnet, M., \& Perrier, C. 1999, A\&A, $341 \mathrm{~L}, 63$

Duquennoy, A., \& Mayor, M. 1991, A\&A, 248, 485

DIVA 1998, http://www.aip.de:8000/ dso/diva

FAME 1998, http://aries.usno.navy.mil/ad/fame/

GAIA 1998, http://astro.estec.esa.nl/SA-general/Projects/GAIA

Hipparcos 1998, http://astro.estec.esa.nl/SA-general/Projects/Hipparcos/

Holman, M., Touma, J., \& Tremaine, S. 1997, Nature, 386, 254

Latham, D.W., Stefanik, R.P., Torres, G., Davis, R.J., Mazeh, T., Carney, B.W., Laird, J.B., \& Morse, J.A. 1999, AJ, submitted

Latham, D.W., Stefanik, R.P., Mazeh, T., Mayor, M., \& Burki, G. 1989, Nature, 339,38

Leung, K.-C., \& Schneider, D.P. 1978, AJ, 83, 618

Marcy, G.W., \& Butler, R.P. 1998, ARA\&A, 36, 57

Marcy, G.W., Butler, R.P., Williams, E., Bildsten, L., Graham, J.R., Ghez, A.M., \& Jernigan, J.G. 1997, ApJ, 481, 926

Mathieu, R.D., Duquennoy, A., Latham, D.W., Mayor, M., Mermilliod, J.-C., \& Mazeh, T. 1992, in Binaries as Tracers of Stellar Formation, A. Duquennoy \& M. Mayor, Cambridge: Cambridge Univ. Press, 278

Mazeh, T., Krymolowski, Y., \& Rosenfeld, G. 1997, ApJ, 477, 103

Rasio, F.A., \& Ford, E.B. 1996, Science, 274, 954

Schneider, J. 1998, http://www.cfa.harvard.edu/planets/

SIM 1998, http://sim.jpl.nasa.gov

Weidenschilling, S.J., \& Marzari, F. 1996, Nature, 384, 619 


\section{Discussion}

Gray: Thanks for the interesting talk. Let me recast the focus and mention one alternative aspect of radial velocities that didn't make it into your talk. There are several astrophysical problems besides planets that can be studied using modern capabilities. If radial velocities of stars are monitored intensively, one might see orbital oscillations that can be subtracted from the signal, allowing one to study interesting things like stellar surface features and non-radial oscillations!

Latham: I didn't try to be all-inclusive. I consciously left out the work on oscillations, knowing that it would come up! 